\title{
ARTICLE
}

Received 27 Mar 2013 | Accepted 5 Aug 2013 | Published 2 Sep $2013 \quad$ DOl: 10.1038/ncomms3404

\section{BRCA1 and CtIP suppress long-tract gene conversion between sister chromatids}

\author{
Gurushankar Chandramouly ${ }^{1}$, Amy Kwok ${ }^{1} \uparrow$, Bin Huang ${ }^{1}$, Nicholas A. Willis ${ }^{1}$, Anyong Xie ${ }^{1} \&$ Ralph Scully $^{1}$
}

BRCA1 controls early steps of the synthesis-dependent strand annealing (SDSA) pathway of homologous recombination, but has no known role following Rad51-mediated synapsis. Here we show that BRCA1 influences post-synaptic homologous recombination events, controlling the balance between short- (STGC) and long-tract gene conversion (LTGC) between sister chromatids. Brca1 mutant cells reveal a bias towards LTGC that is corrected by expression of wild-type but not cancer-predisposing BRCA1 alleles. The LTGC bias is enhanced by depletion of CtIP but reversed by inhibition of 53BP1, implicating DNA end resection as a contributor to the STGC/LTGC balance. The impact of BRCA1/CtIP loss on the STGC/LTGC balance is abolished when the second (non-invading) end of the break is unable to support termination of STGC by homologous pairing (annealing). This suggests that BRCA1/CtIP-mediated processing of the second end of the break controls the annealing step that normally terminates SDSA, thereby suppressing the error-prone LTGC outcome.

\footnotetext{
${ }^{1}$ Beth Israel Deaconess Medical Center and Harvard Medical School, 330 Brookline Avenue, Boston, Massachusetts 02215, USA. ${ }^{\dagger}$ Present address: University of Massachusetts Medical School, 55 Lake Avenue North, Worcester, Massachusetts 01655, USA. Correspondence and requests for materials should be addressed to R.S. (email: rscully@bidmc.harvard.edu).
} 
$\mathrm{D}$ ouble-strand breaks (DSBs) are dangerous DNA lesions, the misrepair of which can promote genomic instability and cancer ${ }^{1,2}$. Chromosome breakage in proliferating cells commonly arises in the $S$ phase of the cell cycle during replication across a damaged DNA template ${ }^{3-5}$. Such DSBs can be repaired by sister chromatid recombination (SCR), a potentially error-free pathway in which the broken chromosome uses the neighbouring sister chromatid as a template for repair by homologous recombination (HR). The major hereditary breast/ovarian cancer predisposition genes, $B R C A 1$ and $B R C A 2$, have established functions in HR. A long-standing hypothesis proposes that BRCA1 and BRCA2 execute tumour suppressor functions in $\mathrm{SCR}^{6}$.

HR entails resection of the DNA end to single-stranded (ss) DNA, on which Rad51 polymerizes to form a Rad51-ssDNA nucleoprotein filament ${ }^{7-9}$. This filament conducts a homology search by invading neighbouring DNA duplexes and base-pairing with a homologous donor such as the neighbouring sister chromatid. A DNA polymerase extends the invading $3^{\prime}$ DNA end (the 'nascent' strand). In somatic eukaryotic cells, the major HR pathway is 'synthesis-dependent strand annealing' (SDSA), a non-crossover pathway in which termination occurs by annealing of the displaced nascent strand with complementary sequences in the resected second end of the broken chromosome ${ }^{7}$. Gene conversion can occur if the homologous donor differs in sequence from the broken chromosome.

Studies in yeast indicate the existence of at least two distinct copying mechanisms in HR, which differ in their fidelity. In Saccharomyces cerevisiae, the majority of somatic gene conversions entail SDSA-mediated copying of a short $(<200 \mathrm{bp})$ stretch of information from the donor. An alternative pathway termed break-induced replication (BIR) can mediate long gene conversions in yeast, potentially extending the nascent strand as far as the end of the chromosome $e^{10-13}$. BIR may entail formation of a bona fide replication fork at the site of recombination and requires both leading and lagging strand synthesis. In yeast, BIR can arise in response to one-ended invasions occurring without a homologous second end, a key trigger being the failure of the second end of the DSB to effect termination of $\mathrm{HR}^{14-17}$. To what extent BIR operates in mammalian cells is not well understood.

In mammalian cells, gene conversions typically extend less than $100 \mathrm{bp}$ ('short-tract' gene conversion-STGC) ${ }^{18-20}$. A small proportion of HR events entail 'long-tract' gene conversion (LTGC), in which nascent strand synthesis extends several kilobases prior to termination ${ }^{21-23}$. LTGC is an error-prone HR outcome, causing tandem gene duplication and, rarely, multicopy gene amplification ${ }^{22}$. Mammalian cells lacking any one of the Rad51 paralogues XRCC3, Rad51C or XRCC2 reveal a specific defect in STGC and marked bias in favour of LTGC, which accounts for $\sim 25 \%$ of all gene conversions in Rad51 paraloguedeficient cells ${ }^{23-25}$. Increased proportions of LTGC-type products were also observed in a $B r c a 2$ mutant hamster cell line and in Nbs1 null chicken DT40 lymphoblastoid cells ${ }^{26,27}$. The identity of other genes that regulate the balance between STGC and LTGC is unknown.

BRCA1 supports DNA end resection via its interactions with CtIP (C-terminus-binding protein of adenovirus E1A-interacting protein) and the Mre11/Rad50/NBS1 (MRN) complex to generate ssDNA that serves as substrate for BRCA2-mediated Rad51 nucleoprotein filament formation ${ }^{28}$. BRCA1 also interacts with BRCA2 via the bridging protein, PALB2 (partner and localizer of BRCA2), as well as with BACH1/BRIP1 and the chromatinassociated RAP80 complex ${ }^{29-32}$. Deletion of 53bp1, a gene implicated in NHEJ and in the suppression of DNA end resection, rescues the Brcal mutant phenotype in the mouse, suggesting a primary role for BRCA1 in DNA end resection ${ }^{33}$.
Thus, the known functions of BRCA1 in HR are restricted to the early steps preceding Rad51-mediated synapsis.

To test whether BRCA1 influences later HR steps, we studied its contribution to STGC and LTGC between sister chromatids, induced by a site-specific chromosomal DSB. We show here that loss of BRCA1 or CtIP skews HR in favour of the LTGC outcome; this is reversed by wild-type BRCA1 but not by certain cancerpredisposing BRCA1 alleles. The influence of BRCA1 and CtIP on the STGC/LTGC balance is lost when the second (non-invading) end of the DSB is unable to support termination of STGC by annealing. We conclude that BRCA1/CtIP controls the balance between STGC and LTGC by acting on the second end of the DSB to support the annealing step that normally terminates STGC. These findings suggest that a defect in the early stages of HR, caused by loss of BRCA1 function, can translate into a defect in HR termination, skewing this process towards error-prone repair at the expense of error-free repair.

\section{Results}

A reporter for rapid flow cytometric analysis of LTGC. We previously described a SCR reporter to simultaneously measure STGC and LTGC between sister chromatids ${ }^{22,34}$. Expression of the rare-cutting homing endonuclease I-SceI ${ }^{35}$ induces a sitespecific DSB within a mutant copy of the gene encoding enhanced green fluorescent protein (E-GFP, here termed 'GFP'). Recombination between the broken GFP copy and neighboring $5^{\prime}$ truncated GFP sequences produces wild-type GFP by gene conversion, and the cell changes from $\mathrm{GFP}^{-}$to $\mathrm{GFP}^{+}$. In the original reporter, duplication of a cassette during LTGC enabled positive selection of LTGC through expression of a wild-type antibiotic resistance gene ${ }^{22}$. We re-fashioned this reporter so that the cassette duplicated during LTGC encodes the monomeric red fluorescent protein RFP1.3 (here termed 'RFP'; Fig. 1a) ${ }^{36}$. Briefly, we divided the RFP cDNA into two artificial exons (' $\mathrm{A}$ ' and ' $\mathrm{B}$ ' in Fig. 1a), with appropriate splice donor and acceptor sequences. The RFP exons were placed 'head to toe' between the two GFP copies of the reporter so that transcription of exon A within the unmodified (parental) reporter will not lead to expression of wildtype RFP. During STGC, the cell becomes $\mathrm{GFP}^{+} \mathrm{RFP}^{-}$(Fig. 1a, outcome no. 1). In contrast, LTGC typically generates three GFP copies (GFP triplication) by SCR and duplicates the RFP cassette (Fig. 1a, outcome no. 2). Splicing between exon A of the first cassette and exon $\mathrm{B}$ of the second generates wtRFP mRNA and the cell becomes $\mathrm{GFP}^{+} \mathrm{RFP}^{+}$. A minority of LTGCs duplicate RFP exon B but terminate prior to triplication of the GFP copies ('early termination' of LTGC-nascent strand extension of between $1,252 \mathrm{bp}$ and $3.4 \mathrm{~kb}$ ).

We targeted a single copy of the RFP-SCR reporter to the ROSA26 locus of the mouse embryonic stem (ES) cell line 11CO/ $47 \mathrm{~T}^{37}$, in which one Brcal allele is truncated and the second can be conditionally inactivated by Cre-mediated recombination (here termed ' $\mathrm{BrCa} 1^{\mathrm{fl} / \mathrm{mut}}$ ' cells, described in detail below; see Methods). Transfection of $\mathrm{Brcal}^{\mathrm{fl} / \mathrm{mut}}$ RFP-SCR reporter cells with I-SceI elicited $\mathrm{GFP}^{+} \mathrm{RFP}^{-}$and $\mathrm{GFP}^{+} \mathrm{RFP}^{+}$products (Fig. 1b). We used fluorescence-activated cell sorting (FACS) to sort single $\mathrm{GFP}^{+} \mathrm{RFP}^{-}$or $\mathrm{GFP}^{+} \mathrm{RFP}^{+}$cells from I-SceItransfected samples, prepared genomic DNA (gDNA) from amplified clones and analysed the structure of the RFP-SCR reporter by Southern blotting. All FACS-sorted $\mathrm{GFP}^{+} \mathrm{RFP}^{-}$ clones revealed a structurally unrearranged RFP-SCR reporter, consistent with STGC (examples in Fig. 1c, lanes G1-G3). In contrast, $>95 \%$ of $\mathrm{GFP}^{+} \mathrm{RFP}^{+}$clones revealed rearrangements of the RFP-SCR reporter characteristic of LTGC (examples in Fig. 1c, lanes T1-T3; more detailed mapping in Supplementary Fig. S1). 
a
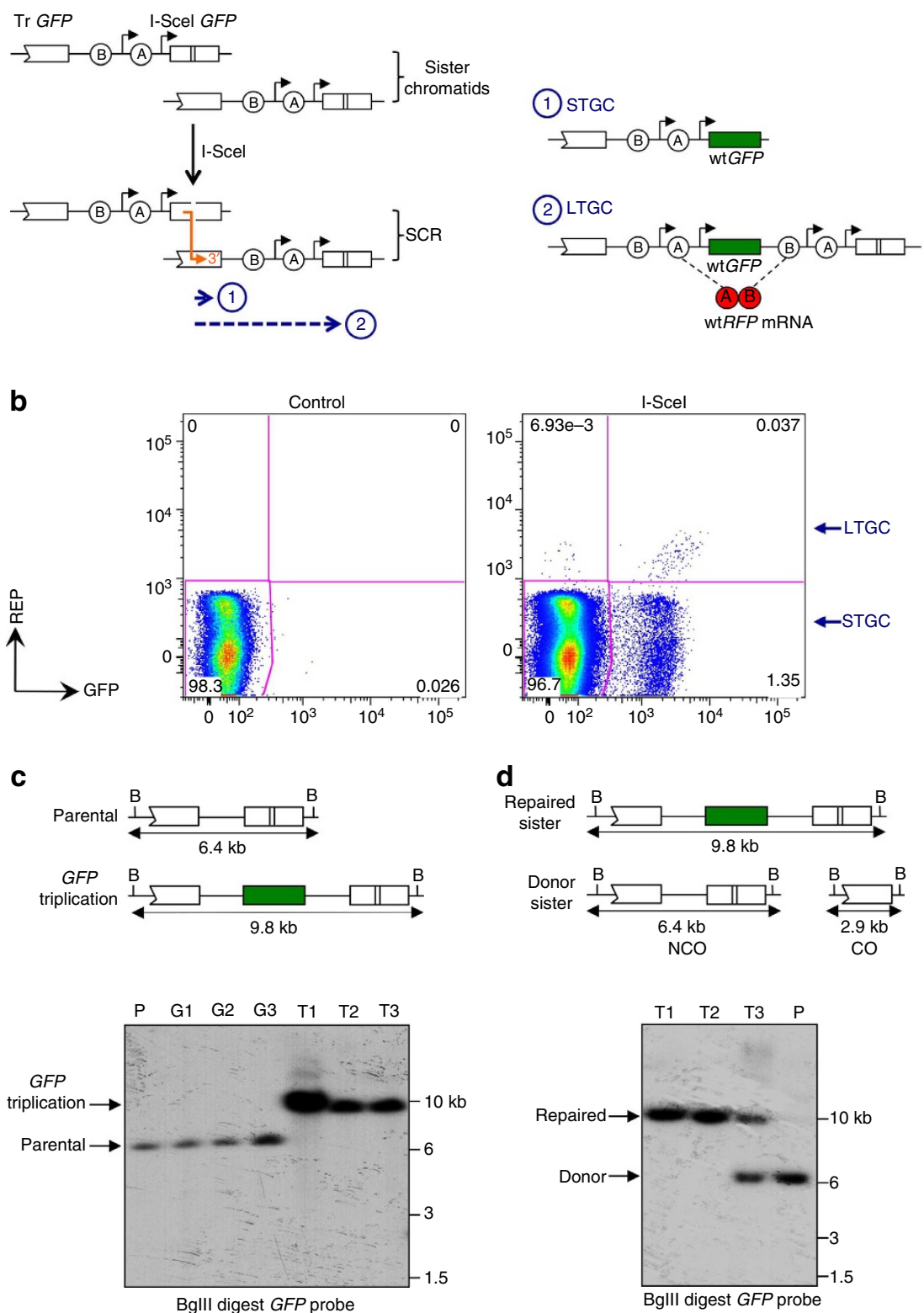

Figure 1 | RFP-SCR reporter for quantifying short-tract and long-tract gene conversion. (a) Structure of the RFP-SCR reporter. Circles A and B:

$5^{\prime}$ and $3^{\prime}$ artificial RFP exons. Black arrows: promoters. Tr GFP: $5^{\prime}$ truncated GFP. Orange arrow: Rad51-mediated strand invasion ( $3^{\prime}$ end marked with arrow head). Blue dashed arrow 1: short-tract gene conversion (STGC); 2: long-tract gene conversion (LTGC). LTGC generates wtRFP mRNA. (b) I-Scel -induced HR products in Brcaf ${ }^{f l / m u t}$ RFP-SCR reporter ES cells. Note GFP ${ }^{+}$RFP $^{-}$(STGC) and GFP ${ }^{+}$RFP $^{+}$(LTGC) populations. (c) Southern blot analysis of HR products. 'Parental': unrearranged RFP-SCR reporter; 'GFP triplication': LTGC or crossover product. B: Bglll sites. Boxes are GFP copies; green box is wtGFP. RFP cassette is not shown. Gel lanes-P: parental; G1-G3: GFP+ RFP' STGC products; T1-T3: GFP+ RFP+ 'GFP triplication' products. (d) Donor sister chromatid structure distinguishes non-crossover (NCO) from crossover (CO) 'GFP triplication' outcome. Cartoon elements as in (c). Gel lanes-T1-T3: GFP ${ }^{+}$RFP ${ }^{+}$clones (different from panel c); P: parental. Note the unrearranged donor sister in T3, implying a NCO mechanism (LTGC).

Donor sister chromatid distinguishes LTGC from crossing over. The 'GFP triplication' outcome could arise by either LTGC or crossing over. In LTGC, the donor sister chromatid is unaltered, whereas crossing over results in loss of one GFP copy from the donor sister (Fig. 1d). Previous work established that crossing over is suppressed in somatic cells ${ }^{21,23}$. To distinguish these mechanisms, we adapted a strategy we used previously to capture the donor sister chromatid in the context of I-SceI-induced $\mathrm{HR}^{23}$. We found that a small fraction of sorted I-SceI-induced GFP ${ }^{+}$ $\mathrm{RFP}^{+}$cells were mixed colonies, in which the GFP ${ }^{+} \mathrm{RFP}^{+}$cell had been sorted subsequent to an LTGC event but prior to the ensuing mitosis. Such clones should therefore contain HR products from both sister chromatids. We identified four such mixed colonies; all (4/4) revealed the 'GFP triplication' outcome and an unrearranged donor (for example, lane T3 in Fig. 1d). Although the number of events detected was small, the consistently unrearranged donor suggests that LTGC is the major mechanism underlying the 'GFP triplication' outcome.

Bias in favour of LTGC in Brcal $^{\Delta / \text { mut }}$ RFP-SCR reporter cells. Figure $2 \mathrm{a}$ depicts the structure of the two Brcal alleles in 
$B r c a 1^{\mathrm{fl} / \mathrm{mut}}$ ES cell line $11 \mathrm{CO} / 47 \mathrm{~T}^{37}$. One allele $\left(B r c a 1^{\mathrm{mut}}\right)$ encodes a C-terminally truncated $\mathrm{Brcal}$ gene product (lacking a functional BRCT repeat). The second allele $\left(B r c a 1^{\mathrm{fl}}\right)$ contains mouse Brcal cDNA sequences corresponding to exons 22-24 flanked by loxP sites (Fig. 2a). Cre-mediated deletion converts the functionally wt $B r c a 1^{\mathrm{fl}}$ allele to a mutant allele $\left(B r c a 1^{\Delta}\right)$ that encodes a C-terminally truncated Brcal product similar to that encoded by the $B r c a 1^{\text {mut }}$ allele. We transduced $B r c a 1^{\mathrm{fl} / \text { mut }}$ RFPSCR reporter ES cells with Cre-encoding adenovirus and screened clones for Brcal inactivation by real-time qPCR (Fig. $2 \mathrm{~b}$ and Methods). We identified Brcal-deleted clones and others that remained undeleted. Brca1 null ES cells are unviable ${ }^{38}$; very likely, $\mathrm{BrCal}^{\text {mut }}$ and $\mathrm{BrCal}^{\Delta}$ are hypomorphic alleles. Consistent with this, we detected by immunoblotting Brcal gene products at low abundance in $\mathrm{Brcal}^{\Delta / \text { mut }}$ ES cell clones. These Brcal proteins were further depleted by siRNA directed to Brcal (Fig. 2b).

To determine whether loss of wtBrcal influences the balance between STGC and LTGC, we transfected, in parallel, the abovenoted Cre-treated $B r C a 1^{\mathrm{fl} / \mathrm{mut}}$ and $\mathrm{BrCa}^{\Delta / \text { mut }}$ RFP-SCR reporter clones with either I-SceI plasmid or empty vector and quantified HR products (Fig. 2c; see Methods). In all experiments described here, clones that were treated with empty vector control revealed typical background levels of $\sim 0.03 \% \mathrm{GFP}^{+} \mathrm{RFP}^{-}$and of $<0.001 \% \mathrm{GFP}^{+} \mathrm{RFP}^{+}$, with at least 200,000 events counted per sample. All I-SceI-induced HR measurements were corrected for background events and for I-SceI transfection efficiency (see Methods), the latter being typically between 65 and 85\%; neither measurement varied with Brcal status. We compared four independent Cre-treated $\mathrm{BrCa}^{\Delta / \mathrm{mut}}$ and $\mathrm{Brcal}^{\mathrm{f} / \mathrm{mut}} \mathrm{RFP}-\mathrm{SCR}$ reporter clones. All four $B r \mathrm{Cal}^{\Delta / \mathrm{mut}}$ clones revealed reduced frequencies of $\mathrm{HR}$, as expected ${ }^{39}$, but only a modest reduction in LTGC (Fig. 2c). The ratio $\mathrm{GFP}^{+} \mathrm{RFP}^{+} /$Total $\mathrm{GFP}^{+}$estimates the probability that an I-SceI-induced $\mathrm{GFP}^{+} \mathrm{HR}$ event will resolve as LTGC. This probability was elevated $\sim 2$-fold in $\mathrm{BrCa}^{\Delta / \mathrm{mut}}$ cells in comparison with isogenic Brcal ${ }^{\mathrm{fl} / \mathrm{mut}}$ cells (Fig. 2c, lower panel). Thus, loss of wtBrcal skews HR in favour of LTGC.

We studied the structure of the reporter in I-SceI-induced $\mathrm{GFP}^{+} \mathrm{RFP}^{+}$clones derived from $\mathrm{Brcal}^{\Delta / \text { mut }}$ cells and isogenic Cre-treated $B r c a 1^{\mathrm{fl} / \mathrm{mut}}$ cells by Southern blotting. As noted above, most LTGCs entail 'GFP triplication' (Fig. 1a, outcome no. 2), while a minority terminate within the reporter subsequent to duplication of $R F P$ exon B ('early termination') ${ }^{21-23,34}$. In $\mathrm{Brcal}^{\mathrm{fl} / \mathrm{mut}}$ cells, 58/65 (89.2\%) $\mathrm{GFP}^{+} \mathrm{RFP}^{+}$clones entailed GFP triplication while $4 / 65(6.1 \%) \mathrm{GFP}^{+} \mathrm{RFP}^{+}$clones revealed early termination of LTGC. In $\mathrm{Brcal}^{\Delta / \mathrm{mut}}$ cells, 28/29 (96.6\%) of $\mathrm{GFP}^{+} \mathrm{RFP}^{+}$clones were GFP triplications and $1 / 29$ (3.4\%) revealed early termination of LTGC. Thus, loss of Brcal does not grossly alter the types of LTGC detected. Capture and analysis of the donor sister chromatid in mixed I-SceI-induced $\mathrm{GFP}^{+} \mathrm{RFP}^{+}$clones sorted from $\mathrm{Brcal}^{\Delta / \mathrm{mut}}$ cells revealed an intact donor sister chromatid in $3 / 3$ GFP triplication clones examined, confirming that they arose by a non-crossover mechanism, that is, by LTGC.
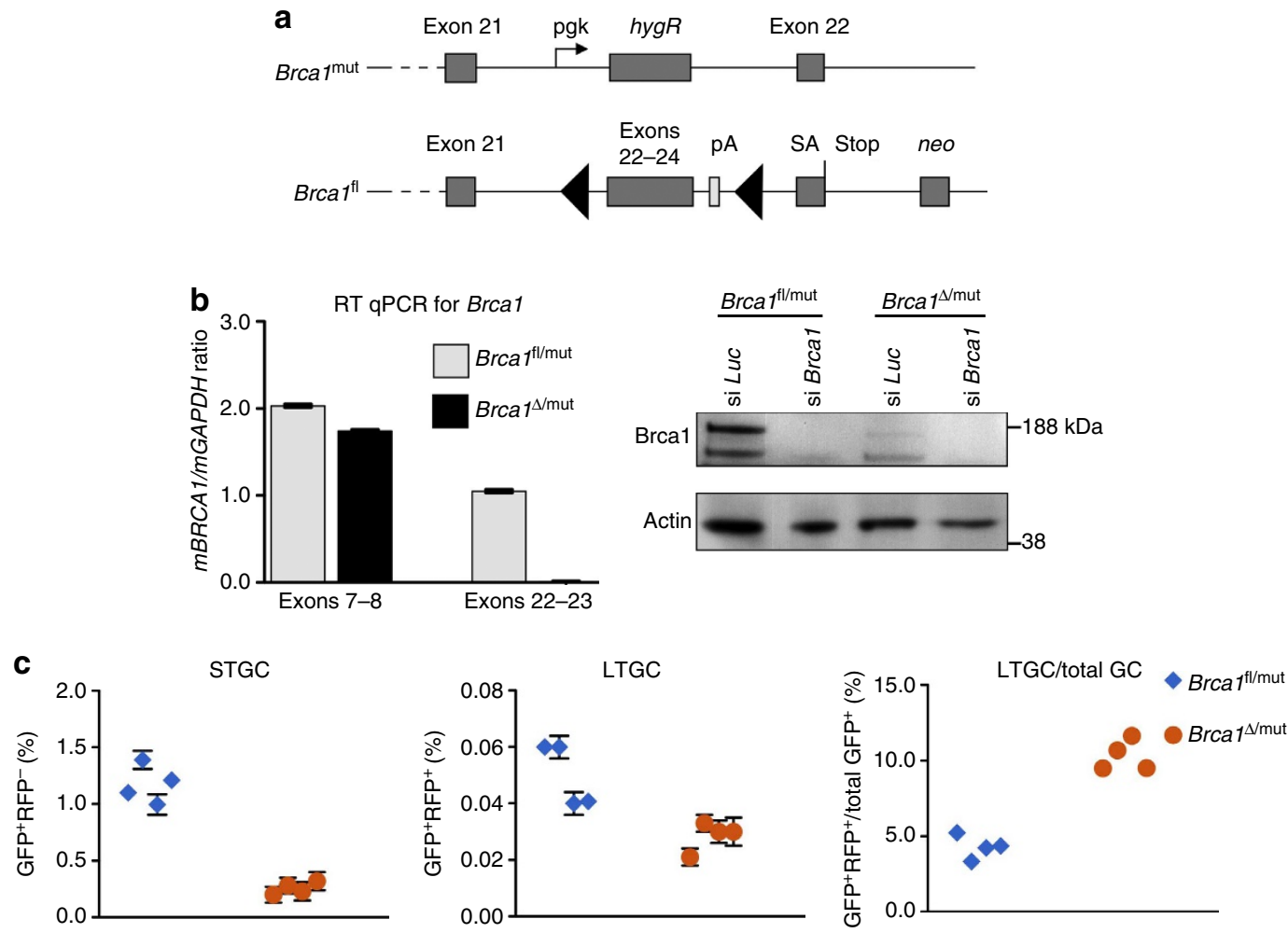

Figure 2 | Bias in favour of LTGC in Brca1 ${ }^{\mathbf{\Delta} / \text { mut }}$ ES cells. (a) Brcal gene structure in Brca $7^{\mathrm{fl} / \text { mut }}$ ES cells. Brca $7^{\text {mut }}$ allele encodes the truncated product. $B r c a 7^{f l}$ allele converts to 'Brca ${ }^{\Delta \prime}$ following Cre-mediated recombination. Grey boxes: Brcal exons; black triangles: loxP sites; SA: splice acceptor. (b) Analysis of Brcal gene products in Brcal fl/mut and Brcal ${ }^{\Delta / \text { mut }}$ ES cells. Left panel: RT qPCR analysis of mRNA exon boundaries. Note the loss of exon $22-23$ signal in Brca ${ }^{\Delta / m u t}$ cells. Right panel: Brca1 protein levels in Brca $7^{\mathrm{fl} / \text { mut }}$ and Brca ${ }^{\Delta / \text { mut }}$ ES cells following transfection with siRNA against luciferase (si Luc) or Brca1 (si Brca1). Note the persistent Brcal product in Brca1 ${ }^{\Delta / \text { mut }}$ ES cells, consistent with hypomorphic status. (c) I-Scel -induced HR in four independent $B r c a 7^{f l / m u t}$ (blue diamonds) and four independent $B r c a 7^{\Delta / m u t}$ (orange circles) Cre-treated RFP-SCR reporter ES cell clones. Each point shows the mean of quadruplicate samples for one independent clone. Error bars: standard error of the mean (s.e.m.) in this and all subsequent figures. STGC, LTGC and LTGC/Total GC products are shown. In the last panel, error bars are smaller than the symbols. 
Wild-type BRCA1 rescues the HR defects of $\mathrm{Brcal}^{\mathrm{\Delta} / \mathrm{mut}}$ cells. The bias in favour of LTGC noted in $B r c a 1^{\Delta / \text { mut }}$ cells could reflect secondary/compensatory responses to Brcal loss. To test this, we transiently co-transfected $B r c a 1^{\Delta / m u t}$ and, in parallel, BrCa1 $1^{\mathrm{fl} / \mathrm{mut}}$ RFP-SCR reporter clones with wt human $B R C A 1$ and I-SceI and measured HR. Human BRCA1 can restore normal development to Brcal null mice ${ }^{40}$. Notably, wild-type BRCA1 complemented the STGC defect and corrected the LTGC bias in $\mathrm{Brca}^{\mathrm{\Delta} / \mathrm{mut}}$ cells, but had little impact on any HR measurements in $\mathrm{Brcal}^{\mathrm{f} / \mathrm{mut}}$ cells (Fig. 3). In contrast to wtBRCA1, expression of cancer-predisposing $B R C A 1$ alleles encoding point mutant products affecting the RING domain (C61G, C64G; Fig. 3a-c) or the tandem BRCT repeat (P1749R, M1775R; Fig. 3d-f) had no impact on HR in Brcal $^{\Delta / \text { mut }}$ cells, despite levels of BRCA1 mutant gene expression higher than those of wtBRCA1 (Supplementary Fig. S2; at the low levels of exogenous $B R C A 1$ used in these experiments, we were unable to detect the hBRCA1 protein). These results suggest that a tumour suppressor function of $B R C A 1$ specifically enforces STGC in favour of LTGC.
The above experiments implicate both the BRCA1 N-terminal RING and C-terminal BRCT domains in STGC and in preventing an LTGC bias during HR. The BRCA1 RING domain mediates constitutive heterodimerization with BARD1 in vertebrate cells and BRCA1 RING domain mutations C61G and C64G disrupt the BRCA1 - BARD1 interaction, while retaining some residual function ${ }^{41,42}$. We used transient transfection of siRNA to deplete either Brcal or Bard1 in $B r c a 1^{\Delta / \text { mut }}$ or $B r c a 1^{\mathrm{fl} / \mathrm{mut}}$ RFPSCR reporter cells in conjunction with I-SceI transfection (see Methods). Consistent with the hypomorphic status of $\mathrm{BrCa}^{\Delta / \text { mut }}$ cells, siRNA-mediated depletion of Brcal reduced HR in both $\mathrm{BrCa}^{\Delta / \mathrm{mut}}$ and $\mathrm{Brcal}^{\mathrm{f} / \mathrm{mut}}$ cells (Supplementary Fig. S3). siRNAmediated depletion of Brcal also further exacerbated the LTGC bias in $\mathrm{BrCa}^{\Delta / \mathrm{mut}}$ cells, but produced only minimal alterations in the STGC/LTGC balance in $B r c a 1^{\mathrm{fl} / \text { mut }}$ cells. A similar pattern was observed following siRNA-mediated depletion of Bard1 (Supplementary Fig. S3). The limited impact of Brca1-specific siRNA on the STGC/LTGC balance in Brcal ${ }^{\mathrm{fl} / \mathrm{mut}}$ cells may reflect the residual activity of wtBrcal in siRNA-depleted a
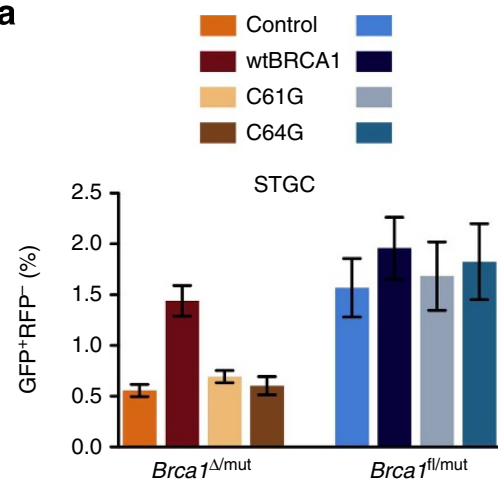

b

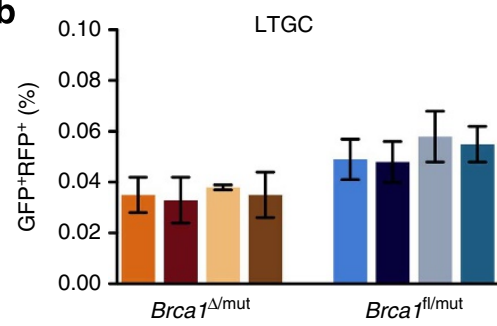

C

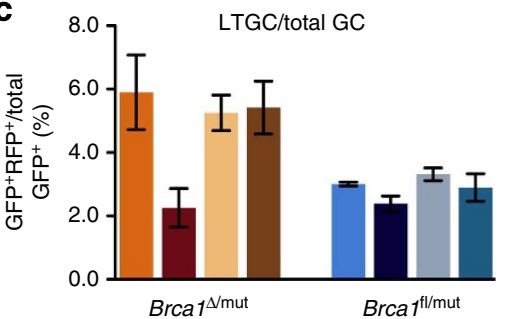

d
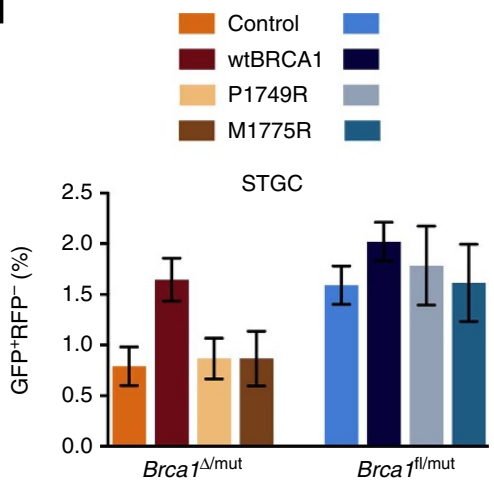

e

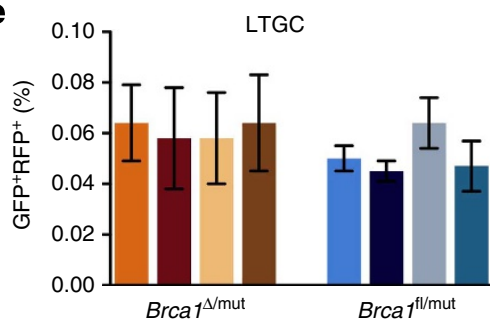

$\mathbf{f}$

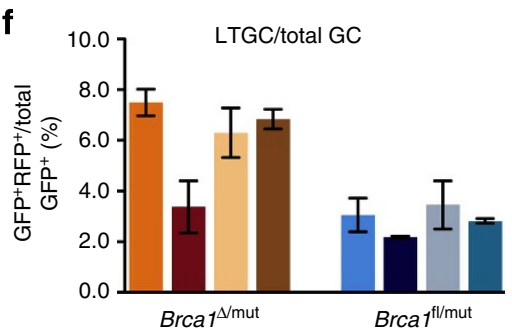

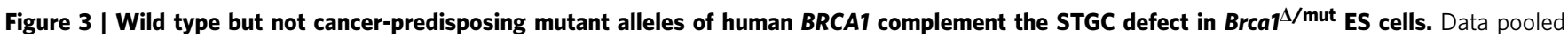
from three independent experiments, each performed with triplicate samples. Error bars: s.e.m., $n=3$. (a) I-Scel -induced STGC in Brcal ${ }^{\Delta / m u t}$ and Brca $7^{\mathrm{fl} / \mathrm{mut}} \mathrm{RFP-SCR}$ reporter ES cells transiently transfected with I-Scel and with control (orange or blue), wtBRCA1 (maroon or dark blue), BRCA1 C61G (apricot or grey) or BRCA1 C64G (brown or teal). Brca1 ${ }^{\Delta / \text { mut }}$ cells: wtBRCA1 versus control: paired $t$-test: $P=0.01$. Other $B R C A 1$ alleles versus control: not significant (NS). Brcaf ${ }^{\mathrm{fl} / \mathrm{mut}}$ cells: all test samples versus control: NS. (b) I-Scel -induced LTGC in the experiment shown in (a). All test samples versus control: NS. (c) Ratio of I-Scel -induced LTGC/overall GC in the experiment shown in (a) and (b). Brcal ${ }^{\Delta / m u t}$ cells: wtBRCA1 versus control: paired $t$-test: $P=0.04$. Other BRCA1 alleles versus control: NS. Brca fl/mut cells: all test samples versus control: NS. (d) Similar to (a), but with BRCA1 P1749R (apricot or grey) or BRCA1 M1775R (brown or teal). Brca1 ${ }^{\Delta / \text { mut }}$ cells: wtBRCA1 versus control: paired $t$-test: $P=0.05$. Other $B R C A 1$ alleles versus control: NS. Brca $7^{\mathrm{fl} / \mathrm{mut}}$ cells: all test samples versus control: NS. (e) I-Scel-induced LTGC events in the experiment shown in (d). All test samples versus control: NS. (f) Ratio of I-Scel-induced LTGC/overall GC in the experiment shown in (d) and (e). Brca ${ }^{1 / \text { mut }^{\prime}}$ cells: wtBRCA1 versus control: paired $t$-test: $P=0.03$. Other BRCA1 alleles versus control: NS. Brca $7^{\mathrm{fl} / \text { mut }}$ cells: all test samples versus control: NS. 
$\mathrm{BrCa1}^{\mathrm{fl} / \mathrm{mut}}$ cells, and indicates that siRNA-mediated methods for perturbing the LTGC/STGC balance have false-negative rates, as was noted previously ${ }^{23}$.

CtIP regulates the balance between STGC and LTGC. At least three major distinct DNA damage response complexes interact with the BRCA1/BARD1 heterodimer via the BRCA1 tandem BRCT repeat, which they bind in a mutually exclusive fashion. These are (direct BRCA1 interactors underlined): CCDC98/ Abraxas, RAP80, BRCC36, BRCC45, and MERIT40; BACH1 and $\overline{\text { TopBP1}}$; and CtIP $^{29-31}$. These interactions are disabled by BRCA1 BRCT mutations P1749R and M1775R. The failure of these BRCA1 BRCT mutants to restore a normal STGC/LTGC balance to $\mathrm{Brcal}^{\Delta / \mathrm{mut}}$ cells therefore suggested possible roles for CtIP, BACH1 or Abraxas/Rap80 as regulators of the STGC/LTGC balance. Bach1 depletion reduced HR in both $\mathrm{BrCa}^{\Delta / \text { mut }}$ and $\mathrm{BrCa1}^{\mathrm{fl} / \mathrm{mut}}$ cells $^{43}$ (Supplementary Fig. S4). This suggests that at least part of Bach1's HR function is independent of its interaction with Brcal. Rap80 depletion caused an unexpected reduction in $\mathrm{HR}$ in both $B r c a 1^{\Delta / \text { mut }}$ and $B r C a 1^{\mathrm{fl} / \text { mut }}$ cells, in contrast to recent findings of several groups in cells with normal BRCA1 function $^{32,44,45}$. We do not understand the reason for this difference; however, Rap80 depletion was found to reduce HR in one other study, suggesting that Rap80's function in HR is contextdependent ${ }^{46}$. siRNA-mediated depletion of CtIP, using either a single siRNA or SMARTpool siRNAs of non-overlapping specificity with the single CtIP siRNA, reduced HR in both $\mathrm{BrCa}^{\Delta / \text { mut }}$ and $\mathrm{Brcal}^{\mathrm{fl} / \mathrm{mut}}$ cells-indicating that CtIP, like Bach1, can function in HR independent of its Brcal interaction. siRNAmediated depletion of $\mathrm{BACH} 1$ or Rap 80 revealed exactly proportional alterations in I-SceI-induced STGC and LTGC in both $B r c a 1^{\Delta / \mathrm{mut}}$ and $B r c a 1^{\mathrm{fl} / \mathrm{mut}}$ cells (Supplementary Fig. S4). In contrast, surprisingly, depletion of CtIP biased HR in favour of LTGC in both $\mathrm{BrCal}^{\Delta / \text { mut }}$ and $\mathrm{BrCal}^{\mathrm{fl} / \text { mut }}$ cells (Fig. $4 \mathrm{a}-\mathrm{c}$ ).

CtIP regulates DNA end resection and is activated by the SIRT6 deacetylase $^{47}$. Notably, Sirt6 depletion mimicked the effect of CtIP depletion, skewing HR in favour of LTGC, as did depletion of Exo1, an exonuclease implicated in DNA end resection (Fig. 5 and Supplementary Fig. S5). Inhibition of 53BP1 - an antagonist of Brcal DNA end resection function ${ }^{33}$ - using a previously characterized dominant-negative fragment of 53BP1 (ref. 48), specifically restored the STGC/LTGC balance to $\mathrm{Brcal}^{\Delta / \text { mut }}$ cells but did not affect this balance in $B r c a 1^{\mathrm{fl} / \text { mut }}$ cells (Fig. 5). This supports the idea that the LTGC bias in $\mathrm{Brcal}^{\Delta / \text { mut }}$ cells is caused by a defect in DNA end resection. siRNA-mediated depletion of the early DSB response protein Mre11 had no impact on the STGC/LTGC balance, suggesting that not all resection proteins a

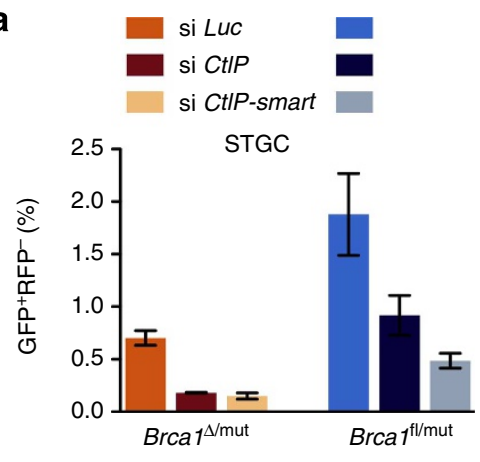

b

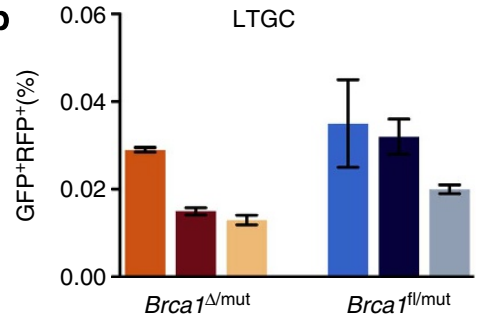

C

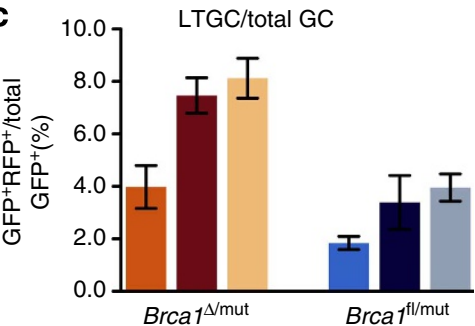

d

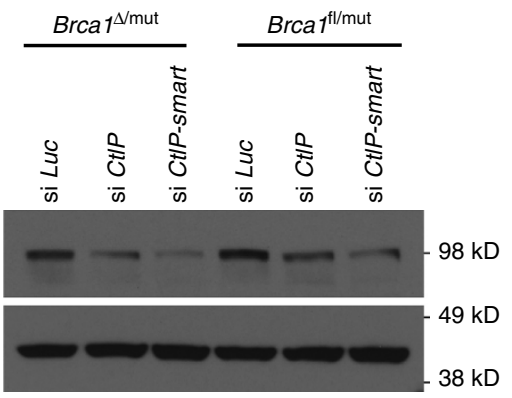

e
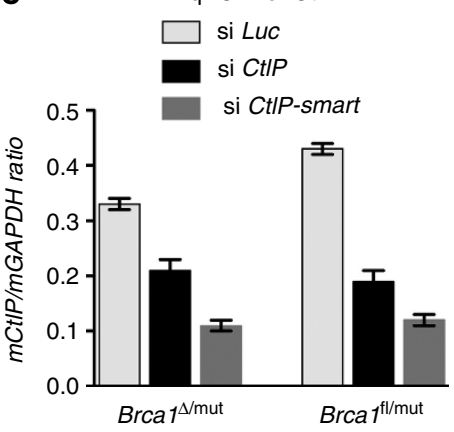

Figure 4 | CtIP controls the balance between LTGC and STGC. Data pooled from three independent experiments, each performed with triplicate samples. Error bars: s.e.m., $n=3$. (a) I-Scel-induced STGC in Brcal ${ }^{\Delta / m u t}$ and Brca $7^{f l / m u t}$ RFP-SCR reporter ES cells co-transfected with I-Scel and with control Luciferase siRNA (si Luc, orange or blue), single CtIP siRNA (si CtIP, maroon or dark blue) or CtIP SMARTpool (si CtIP-smart, apricot or grey). Paired t-test versus si Luc in Brca1 ${ }^{\Delta / \text { mut }}$ cells-si CtIP: $P=0.03$; si CtIP-smart: $P=0.03$; in Brca $7^{f / / m u t}$ cells-si CtIP: NS. si CtIP-smart: $P=0.05$. (b) I-Scel-induced

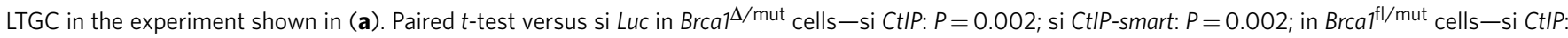
NS. si CtIP-smart: NS. (c) Ratio of I-Scel-induced LTGC/overall GC in the experiment shown in (a) and (b). Paired $t$-test versus si Luc in Brca ${ }^{\Delta / \text { mut }}$ cells-si CtIP: $P=0.04$; si CtIP-smart: $P=0.04$; in Brca ${ }^{f l / m u t}$ cells-si CtIP: NS. si CtIP-smart: $P=0.01$. (d) Abundance of CtIP and actin (loading control) in the siRNA-treated cells used in (a), (b) and (c). (e) RT qPCR analysis of CtIP mRNA in the siRNA-treated cells used in panels (a), (b) and (c). 


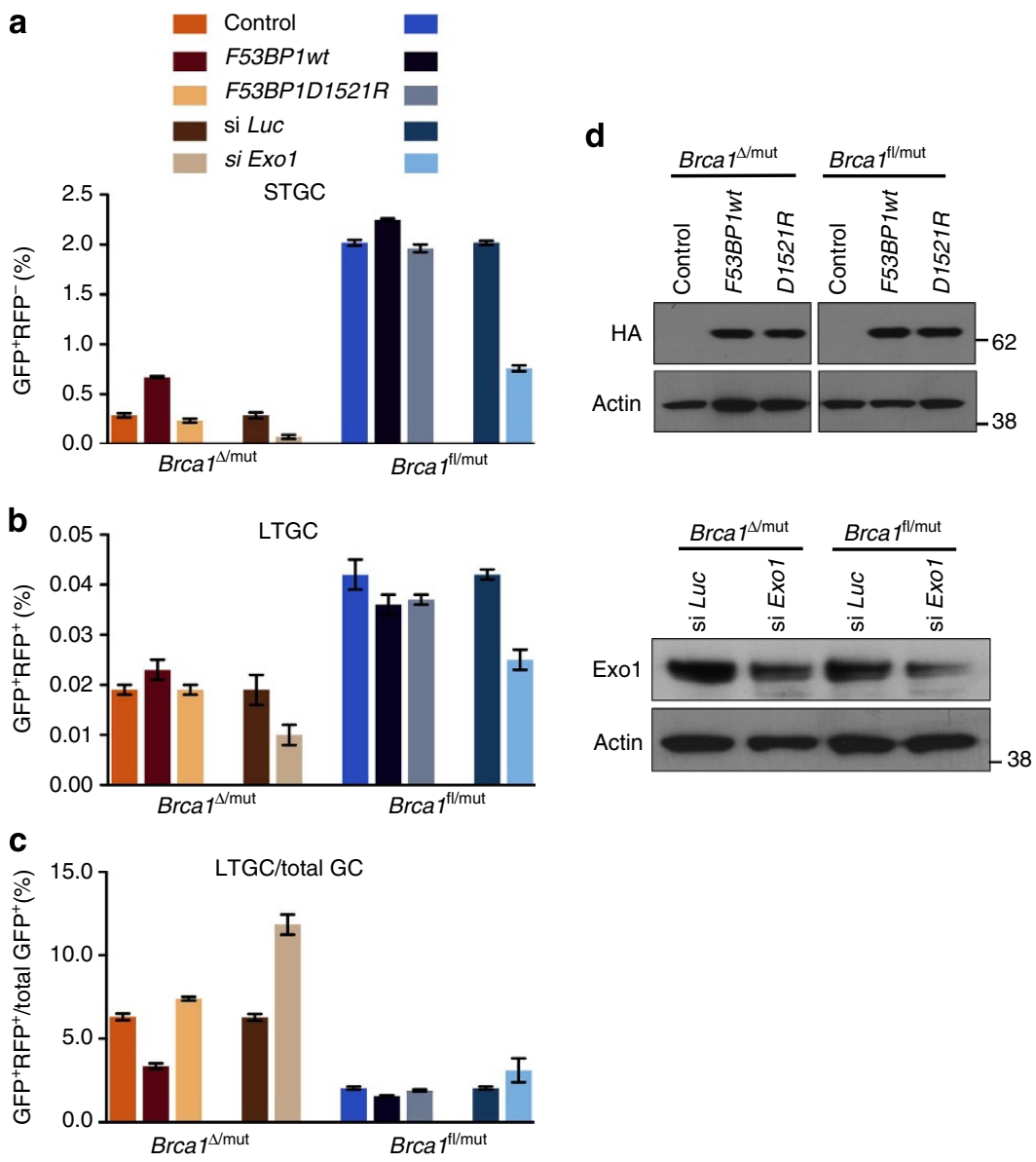

Figure 5 | Impact of overexpression of 53BP1 fragment and siRNA-mediated depletion of Exo1 on STGC and LTGC. Data pooled from three independent experiments, each performed with triplicate samples. Error bars: s.e.m., $n=3$. (a) I-Scel-induced STGC frequencies in Brca7 ${ }^{\Delta / \mathrm{mut}}$ and Brca7 ${ }^{\mathrm{fl} / \mathrm{mut}}$ RFP-SCR reporter ES cells transiently co-transfected with I-Scel expression vector and with control vector (Control, orange or blue), F53BP1wt fragment (F53BP1wt, maroon or dark blue), F53BP1D1521R fragment (D1521R, apricot or grey), Luciferase control siRNA (si Luc, brown or teal) or SMARTpool siRNA against Exo1 (si Exo1, light brown or sky blue). Paired t-test versus control in Brca ${ }^{\Delta / m_{u t}}$ cells-F53BP1wt: $P=0.0001, D 1521 R$ : NS; in Brcafl/mut cellsF53BP1wt: NS, D1521R: NS. Paired $t$-test si Luc versus si Exo1 in Brca ${ }^{\Delta / m u t}$ cells: $P=0.00007$, in Brca $7^{f l / m u t}$ cells: $P=0.0001$. Error bars indicate s.e.m. throughout. (b) Frequency of I-Scel-induced LTGC events in the same experiment as in panel (a). Paired $t$-test versus control in Brca ${ }^{\Delta / \text { mut }}$ cells $-F 53 B P 1 w t$ :

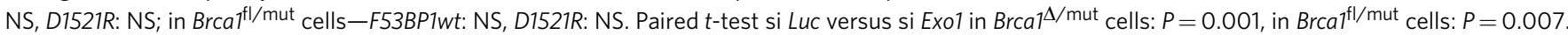
(c) Ratio of I-Scel-induced GFP ${ }^{+} \mathrm{RFP}^{+}$/total GFP ${ }^{+}$frequencies (LTGC/overall GC, expressed as a percentage) from the same experiment as in panels (a) and (b). Paired $t$-test against control in Brca ${ }^{\Delta / \text { mut }}$ cells-F53BP1wt: $P=0.0003$, D1521R: NS; in Brcafl/mut cells-F53BP1wt: NS, D1521R: NS. Paired $t$-test si $L$ L versus si Exo1 in Brca ${ }^{\Delta / \text { mut }}$ cells: $P=0.0001$, in Brca ${ }^{f l / m u t}$ cells: $P=0.007$. (d) Abundance of 53BP1 fragments, Exo1 and actin (loading control) in treated $\mathrm{Brca}^{\Delta / \mathrm{mut}}$ and $B r c a 7^{\mathrm{fl} / \mathrm{mut}} \mathrm{RFP}-\mathrm{SCR}$ reporter ES cells in (a), (b) and (c).

influence this balance (Supplementary Fig. S5). We considered the possibility that the LTGC bias in ES cells depleted of CtIP might reflect altered cell cycle distribution ${ }^{49}$. However, cell cycle distribution did not vary between $B r c a 1^{\Delta / \text { mut }}$ or $B r c a 1^{\mathrm{fl} / \mathrm{mut}}$ cells, whether they received siRNA directed to Luciferase, Bard1 or CtIP (Supplementary Fig. S6). We determined whether the LTGC bias in cells lacking BRCA1/BARD1/CtIP is unique to mouse ES cells by studying human U2OS osteosarcoma cells carrying a single integrated copy of the RFP-SCR reporter (see Methods). Consistent with the above results, we observed a bias towards LTGC in U2OS cells depleted of BRCA1, BARD1 or CtIP (Supplementary Fig. S7). Taken together, the results reveal a surprising and specific role for BRCA1/BARD1, CtIP and certain other DNA end resection complexes in controlling the balance between STGC and LTGC.

Brca1/CtIP controls the annealing step of SDSA. The idea that a defect in DNA end resection could translate into a defect in late stages of HR seems paradoxical. We considered the hypothesis that the bias towards LTGC in Brcal mutant cells is the consequence of failed termination of STGC. We tested this hypothesis by constructing a new 'one-ended' RFP-SCR reporter, in which the non-invading DNA end (marked with a red star in Fig. 6a) lacks GFP sequences. I-SceI -induced STGC $\left(\mathrm{GFP}^{+} \mathrm{RFP}^{-}\right)$ occurring in this reporter cannot be terminated by homologous pairing (annealing), but must instead use non-homologous mechanisms to rejoin the displaced nascent strand with the noninvading, non-homologous second end of the DSB.

We targeted a single copy of the 'one-ended' RFP-SCR reporter to the ROSA26 locus of $B r c a 1^{\mathrm{fl} / \mathrm{mut}}$ ES cells. We generated isogenic clones of Cre-treated $\mathrm{BrCal}^{\mathrm{fl} / \mathrm{mut}}$ one-ended RFP-SCR reporter cells that either had or had not undergone deletion of wtBrca1. Consistent with the longer gene conversion (332 bp) required to produce $\mathrm{GFP}^{+}$products, we noted $\sim 10$-fold lower frequencies of I-SceI -induced STGC $\left(\mathrm{GFP}^{+} \mathrm{RFP}^{+}\right)$products in Brcal $1^{\mathrm{fl} / \mathrm{mut}}$ cells compared to isogenic $B r c a 1^{\mathrm{fl} / \mathrm{mut}}$ cells containing a conventional 'two-ended' RFP-SCR reporter (Fig. 6b); in 
contrast, the absolute frequencies of I-SceI-induced LTGC were little changed (Fig. 6b). Typically, about one-third of all measured HR products were $\mathrm{GFP}^{+} \mathrm{RFP}^{+}$, the remainder being $\mathrm{GFP}^{+} \mathrm{RFP}^{-}$ ; this varied from clone to clone, the maximum LTGC:total HR ratio being $\sim 60 \%$. Southern analysis of FACS-sorted I-SceIinduced $\mathrm{GFP}^{+} \mathrm{RFP}^{-}$or $\mathrm{GFP}^{+} \mathrm{RFP}^{+}$populations derived from either $B r c a 1^{\mathrm{fl} / \mathrm{mut}}$ or from $B r c a 1^{\Delta / \text { mut }}$ cells revealed STGC products of variable size, reflecting random termination by end joining (Fig. 6c); LTGC products revealed a combination of 'early terminating' LTGC (that is, LTGC with termination occurring between the two GFP copies of the donor) and 'GFP triplications' (Fig. 6c). Consistent with the absence of an annealing step that
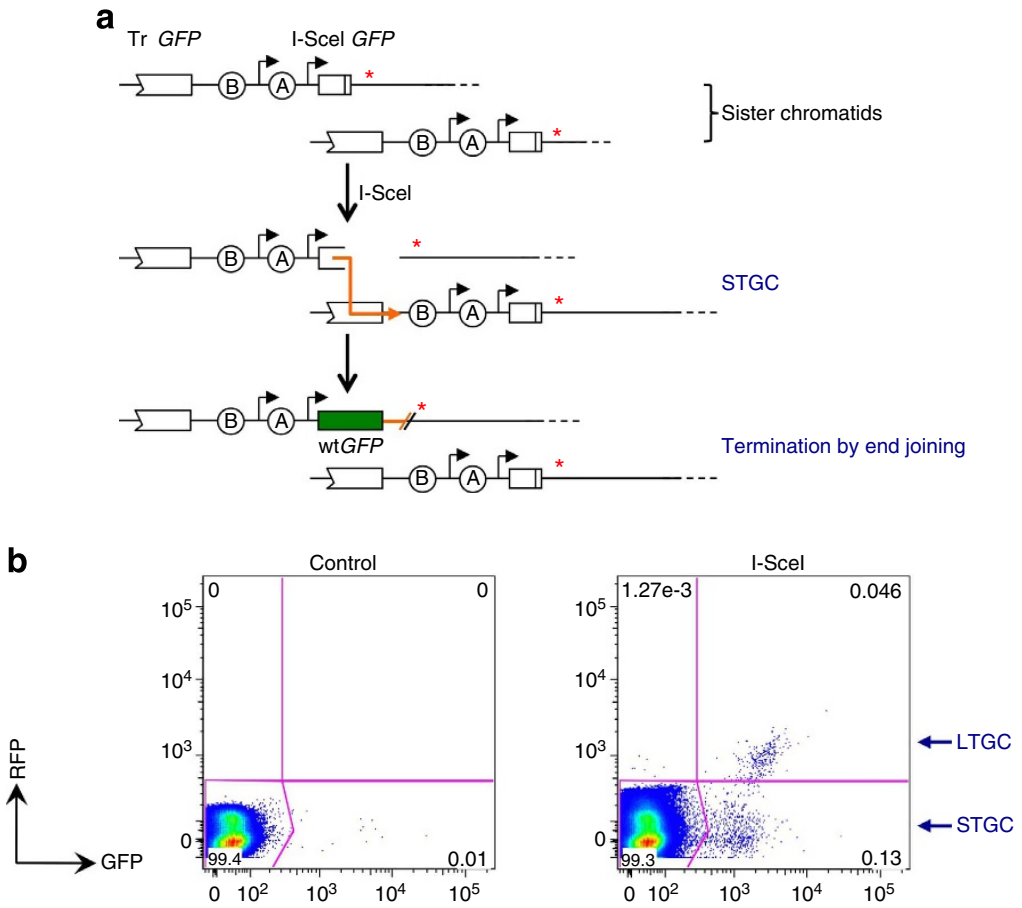

c
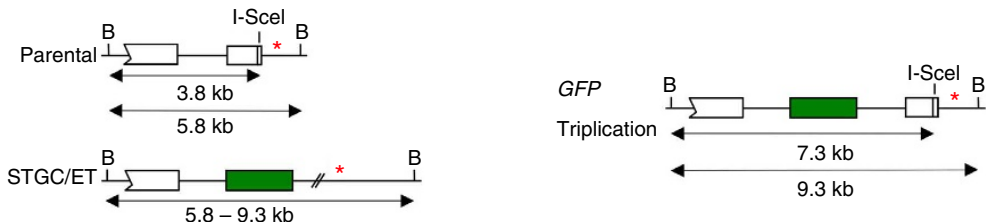

Brca $1^{\text {fl/mut }}$

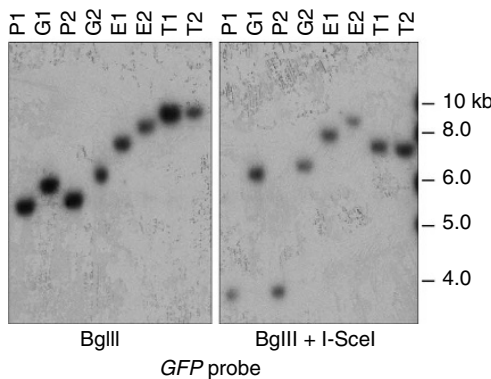

Brca1 $1^{\mathrm{N} \text { mut }}$

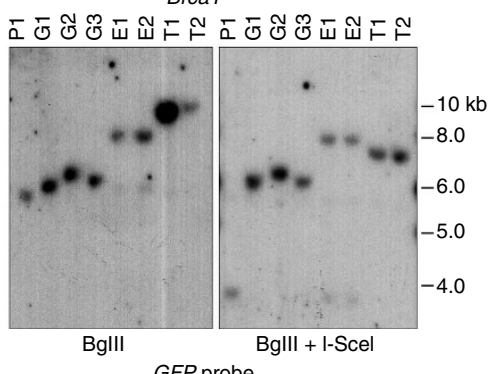

Figure 6 | Analysis of I-Scel-induced STGC and LTGC in 'one-ended' HR reporter cells. (a) Structure of the 'one-ended' RFP-SCR reporter. Circles A and B: $5^{\prime}$ and $3^{\prime}$ artificial RFP exons. Tr GFP: $5^{\prime}$ truncated GFP. Black arrows: promoters. Orange arrow: Rad51-mediated strand invasion (3' end marked with arrow head). The second (non-invading) end of DSB lacks GFP sequences (marked with red star; compare with Fig. 1a). The annealing step is not available to terminate STGC $\left(\mathrm{GFP}^{+} \mathrm{RFP}^{-}\right)$and STGC termination must occur by end joining. $\mathrm{LTGC}\left(\mathrm{GFP}^{+} \mathrm{RFP}^{+}\right)$products are not shown. (b) Primary FACS data showing I-Scel-induced HR products in Brcal ${ }^{\mathrm{fl} / \text { mut }}$ 'one-ended' RFP-SCR reporter ES cells. Note the I-Scel-induced GFP ${ }^{+} \mathrm{RFP}^{-}$(STGC) and GFP+ RFP ${ }^{+}$ (LTGC) populations. (c) Southern blot analysis of HR products in one-ended RFP-SCR reporter cells. 'Parental': unrearranged reporter; 'ET': 'Early termination' LTGC products. For 'STGC/ET' events, termination is predicted to occur by end-joining, producing HR products of varying size. 'GFP triplication': LTGC product. B: BgI II sites. I-Scel sites are marked, as are restriction fragment sizes. Red star: non-homologous second end of I-Scel-induced DSB. Boxes are GFP copies; the green box is wtGFP. RFP cassette is not shown. gDNA from individual clones was digested in vitro with Bglll alone or with BgllI + I-Scel as shown; Southern blot membranes were probed with GFP probe. The two left-hand panels (Brca ${ }^{\mathrm{fl} / \mathrm{mut}}$ ) are taken from one gel. The two right-hand panels (Brca1 ${ }^{\Delta / \text { mut }}$ ) are taken from one gel. Gel lanes-P1-P2: parental clones; G1-G3: STGC (GFP+ RFP') clones; E1-E2: early termination LTGC $\left(\mathrm{GFP}^{+} \mathrm{RFP}^{+}\right)$clones; T1-T2: $\left.\mathrm{LTGC}_{(\mathrm{GFP}}{ }^{+} \mathrm{RFP}^{+}\right)$'GFP Triplication' clones. Only 'parental' and 'GFP triplication' clones contain an I-Scel site within the repaired reporter; this site is lost in the STGC and ET clones. The slight differences in gel mobility of the G1, G2, E1 and E2 bands between the two panels from the Brcafl/mut gel reflect bowing of the gel during electrophoresis or Southern transfer. 
a
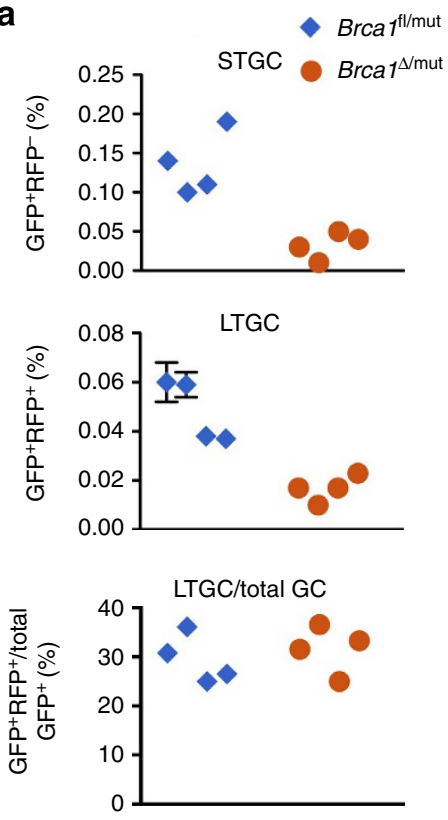

b

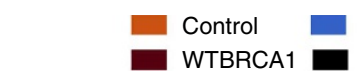

WTBRCA1

STGC
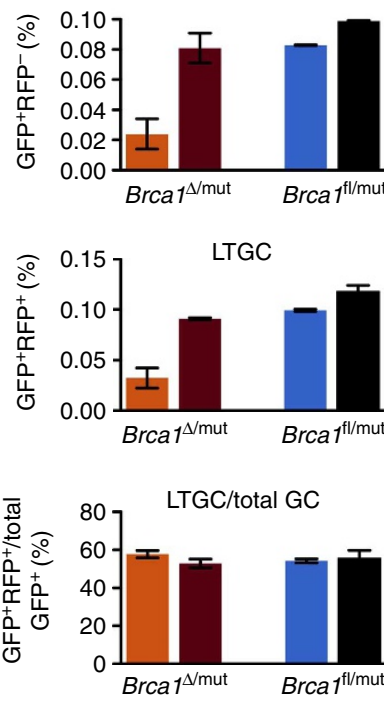

C $\square$ si Luc $\square$

STGC
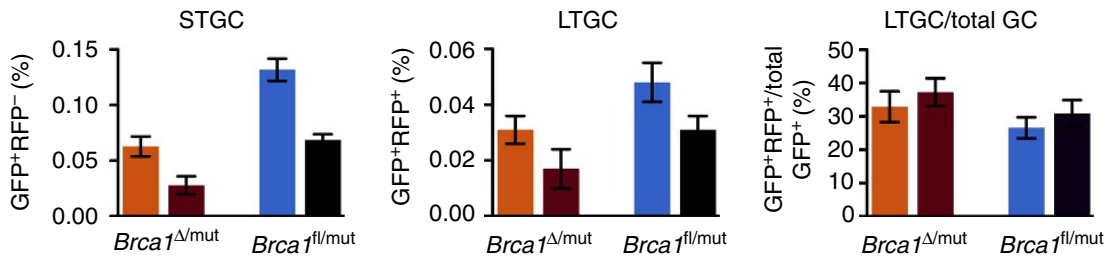

Figure 7 | BRCA1/CtIP fails to affect the STGC/LTGC balance if the annealing step of STGC termination is abolished. (a) I-Scel-induced HR in four independent Cre-treated one-ended RFP-SCR reporter ES cell clones of each genotype: Brca $7^{\mathrm{fl} / \mathrm{mut}}$ (blue diamonds) and Brca $7^{\Delta / \mathrm{mut}}$ (orange circles). Each point shows the mean of quadruplicate samples for one independent clone. Error bars: standard error of the mean (s.e.m.). If invisible, error bars are smaller than symbols. (b) Wild-type human BRCA1 complements both the STGC and LTGC defects in Brca ${ }^{\Delta / \text { mut }}$ one-ended RFP-SCR reporter ES cells. Data pooled from three independent experiments, each performed in triplicate. Error bars: s.e.m., $n=3$. Top panel: I-Scel-induced STGC in Brca1 ${ }^{\Delta / \text { mut }}$ and $B r c a 7^{f l / m u t}$ one-ended RFP-SCR reporter ES cells transiently transfected with I-Scel and with either control (orange or blue) or wtBRCA1 (maroon or dark blue) expression vectors. Brca ${ }^{\Delta / \text { mut }}$ cells: wtBRCA1 versus control: paired $t$-test: $P=0.003$. Brca $7^{f l / m u t}$ cells: wtBRCA1 versus control: NS. Middle panel: I-Scel-induced LTGC in the experiment shown in the top panel. Brca ${ }^{\Delta / \text { mut }}$ cells: wtBRCA7 versus control: paired t-test: $P=0.003$. Brca ${ }^{f / / m u t}$ cells: wtBRCA1 versus control: NS. Bottom panel: Ratio of I-Scel-induced LTGC/overall GC in the experiment shown in the upper panels. Brca ${ }^{\Delta / \text { mut }}$ cells: wtBRCA1 versus control: NS. Brcaf ${ }^{f l / m u t}$ cells: wtBRCA1 versus control: NS. (c) CtIP depletion reduces STGC and LTGC in equal proportions in one-ended RFP-SCR reporter cells. Data pooled from three independent experiments, each performed with triplicate samples. Left panel: I-Scel-induced STGC in Brca ${ }^{\Delta / \text { mut }}$ and Brcafl/mut RFP-SCR reporter ES cells co-transfected with I-Scel and with either control Luciferase siRNA (si Luc, orange or blue bars) or CtIP SMARTpool (si CtIP, maroon or dark blue bars). Paired $t$-test in Brca ${ }^{\Delta / \text { mut }}$ cells: $P=0.05$; in Brcaf ${ }^{f / / m u t}$ cells: $P=0.02$. Middle panel: I-Scel-induced LTGC in the same experiment. Paired $t$-test $B r c a 7^{\Delta / m u t}$ cells: $P=0.04$; in Brca $7^{\mathrm{fl} / \text { mut }}$ cells: NS. Right panel: Ratio of I-Scel-induced LTGC/overall GC in the same experiment. Paired $t$-test in $\mathrm{Brca}^{\Delta / \mathrm{mut}}$ cells: NS; in Brca $7^{\mathrm{fl} / \mathrm{mut}}$ cells: NS.

can reliably terminate STGC, we noted a higher proportion of 'early terminating' LTGCs in one-ended reporter cells than in the original two-ended reporter cells: in $\mathrm{BrCal}^{\mathrm{fl} / \mathrm{mut}}$ one-ended RFP-SCR reporter cells, 6/21 (29\%) of all LTGCs were early terminating and $15 / 21$ (71\%) were GFP triplications; in $\mathrm{BrCa1}^{\Delta / \mathrm{mut}}$ one-ended RFP-SCR reporter cells, 9/23 (39\%) of all LTGCs were early terminating and 14/23 (61\%) were GFP triplications (difference between $\mathrm{BrCa}^{\mathrm{fl} / \mathrm{mut}}$ and $\mathrm{BrCa}^{\Delta / \mathrm{mut}}$ is not significant by $\chi^{2}$ analysis).

We noted reduced I-SceI-induced HR frequencies in four independent one-ended RFP-SCR reporter $B r c a 1^{\Delta / \text { mut }}$ clones, in comparison with four independent $B r c a 1^{\mathrm{l} / \mathrm{mut}}$ clones (Fig. 7a). However, HR in $\mathrm{Brcal}^{\Delta / \text { mut }}$ one-ended RFP-SCR reporter cells revealed exactly proportionate reductions in STGC and LTGC, with no additional LTGC bias (Fig. 7a). Further, transient expression of wtBRCA1 in Brcal $1^{\Delta / \text { mut }}$ one-ended RFP-SCR reporter cells restored both STGC and LTGC in equal proportions (Fig. 7b). Thus, in contrast to the conventional two-ended RFP-SCR reporter (Fig. 3), in the context of the one-ended RFP-SCR reporter, STGC and LTGC are each equivalently Brca1-dependent (Fig. 7b). Further, siRNA-mediated depletion of CtIP reduced STGC and LTGC in equal proportions in both $B r c a 1^{\mathrm{f} / \mathrm{mut}}$ and $\mathrm{Brcal}^{\Delta / \mathrm{mut}}$ one-ended RFP-SCR reporter cells and thus had no statistically significant impact on the STGC/ LTGC balance (Fig. 7c). Therefore, when the annealing step of STGC is inactivated, loss of Brcal/CtIP has no impact on the relative balance between STGC and LTGC.

\section{Discussion}

This report documents a functional communication between BRCA1 and later stages of HR, as revealed by a bias towards 
LTGC when BRCA1 function is impaired. Expression of wildtype but not cancer-predisposing $B R C A 1$ alleles restored the STGC/LTGC balance in Brcal mutant cells, suggesting that $B R C A 1$ performs a tumour suppressor function in this process. Depletion of the BRCA1-interacting end resection protein CtIP (or its activator, Sirt6) or of Exol mimicked or enhanced the Brcal mutant STGC/LTGC imbalance. Further, inhibition of 53BP1, a key antagonist of the DNA end resection function of Brca1, normalized the STGC/LTGC balance in $\mathrm{Brcal}^{\Delta / \mathrm{mut}}$ cells. This suggests that defects in DNA end processing are the cause of the LTGC bias in $\mathrm{BrCal}^{\Delta / \mathrm{mut}}$ cells. To identify the mechanisms underlying this phenomenon, we studied STGC and LTGC within a new 'one-ended' reporter, in which STGC cannot be terminated by annealing. Surprisingly, in the context of the 'oneended' reporter, although overall HR retained dependence on BRCA1 and CtIP, loss of BRCA1/CtIP no longer influenced the balance between STGC and LTGC. This suggests that BRCA1/ CtIP influences the STGC/LTGC balance in the context of twoended DSBs by facilitating the annealing step that normally terminates STGC-most likely, by ensuring efficient and timely processing of the second end of the DSB (Fig. 8).

The SDSA model of somatic HR assumes an asymmetry between the two DNA ends, such that one end undergoes Rad51mediated strand exchange and the second end does not (Fig. 8) ${ }^{7}$. How asymmetry is established during SDSA is unknown; it could be imposed at several different stages, including the DNA end resection stage or during Rad51 filament assembly. Although the model proposed in Fig. 8 envisions a direct role for Brcal, CtIP and Exo1 in resection of the non-invading second end of the DSB, one or more of these proteins might also coordinate communication between the two DNA ends during end resection. Interestingly, recent work in Saccharomyces cerevisiae shows that the Mre11 nuclease and the CtIP homologue, Sae2, provide coordinated resection of the two ends of radiation-induced $\mathrm{DSBs}^{50}$. Currently, however, it is not possible to measure secondend resection or potential asymmetry between the two DNA ends in mammalian cells. Interestingly, the failure of Mre11 depletion to influence the STGC/LTGC balance suggests that not all mammalian complexes implicated in DNA end resection affect the balance between STGC and LTGC. In this regard, deletion of $H 2 A X$, an HR gene that can suppress DNA end resection in certain contexts $^{51}$, does not appear to influence the STGC/LTGC balance $^{34}$.

Analyses of the impact of POL32 mutation on gap repair in $S$. cerevisiae ${ }^{52}$ and in Drosophila melanogaster ${ }^{53}$ suggest that SDSA may entail several different mechanisms of nascent strand extension that differ in their processivity and, hence, in their capacity to mediate LTGC. A major alternative model proposes that mammalian LTGC is mediated by a distinct copying mechanism, such as BIR. BIR may entail formation of a bona fide replication fork following strand exchange and therefore involves lagging strand synthesis ${ }^{11,15,54}$. A key trigger to BIR in yeast is a one-ended invasion occurring without a homologous second end ${ }^{10,11,14-17}$. In yeast, both RAD51-independent and RAD51-dependent forms of BIR are recognized ${ }^{7,11,13-15}$. RAD51independent BIR can act on short homologous sequences, tolerating homologies down to $\sim 30 \mathrm{bp}^{55}$. By analogy, a defect in DNA end resection and Rad51 loading in Brcal mutants might lead to the formation of abnormal synapses that favour BIRperhaps similar to the recently proposed idea of microhomologymediated BIR (MM-BIR) ${ }^{56}$-leading to the observed LTGC bias in Brcal mutants. We consider this BIR model of LTGC in detail below.

In contrast to the avid engagement of BIR following chromosomal one-ended invasions in S. cerevisiae, the majority of chromosomal one-ended HR events in the mammalian system a

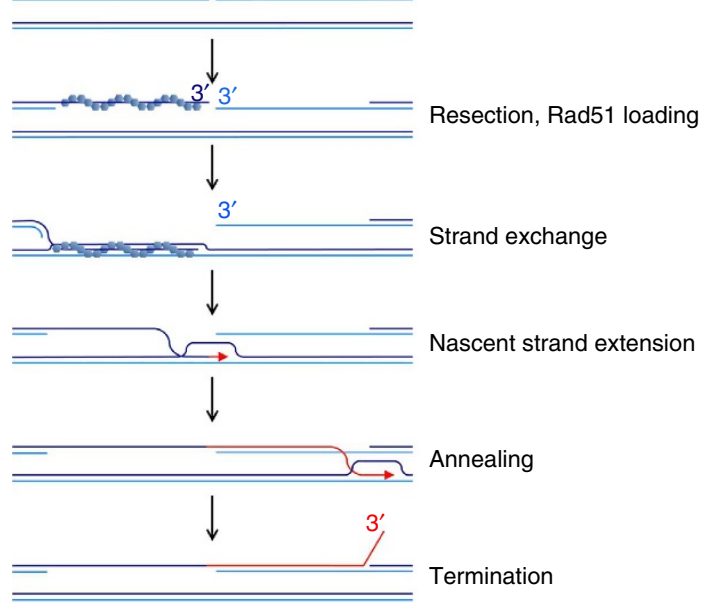

b

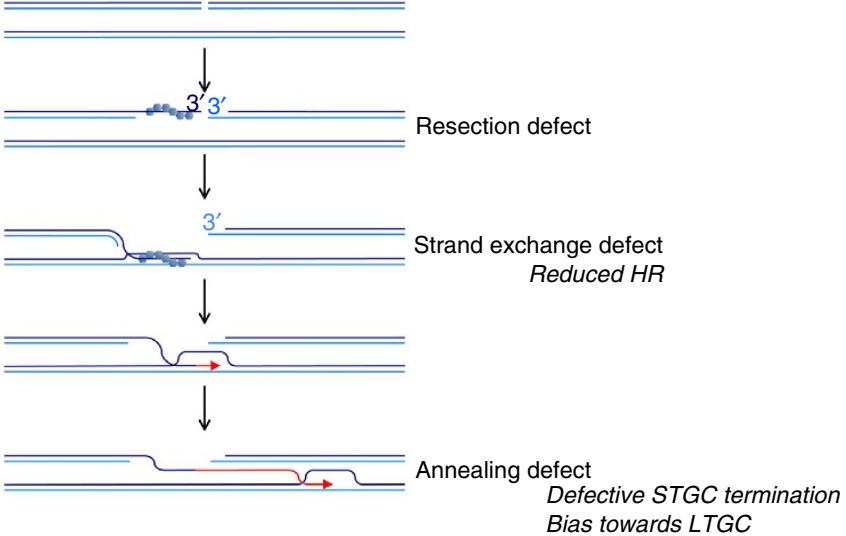

Figure 8 | Model: Brca1/CtIP acts on the second end of the DSB to promote the annealing step of SDSA. (a) SDSA in wild-type cells. Structural analysis of STGC in Figs 1c and 6c suggests that the homologous second end of the DSB normally 'edits' short-tract gene conversions to a defined size. (b) In cells lacking Brca1/CtIP function, defective DNA end resection and impaired cooperation with Brca2/Rad51 results in a strand exchange defect. A defect in second-end resection leads to a relative impairment in the annealing step that normally terminates STGC. As a result, a higher proportion of HR events escape termination as STGCs and resolve as LTGCs. In the absence of a homologous second end of the DSB (such as in the 'one-ended' reporter-not shown in this figure), loss of Brca1/CtIP function has no impact on STGC termination and does not affect the STGC/LTGC balance.

studied here resolve as STGCs, as revealed by the abundance of $\mathrm{GFP}^{+} \mathrm{RFP}^{-}$products in this setting. There is indeed a proportionate bias in favour of LTGC in the 'one-ended' reporter compared to the 'two-ended' reporter; however, the absolute frequencies of I-SceI -induced LTGC at the ROSA26 locus in $B r c a 1^{\mathrm{fl} / \mathrm{mut}}$ cells are equal in the two reporters $(\sim 0.05 \%$; compare the LTGC frequencies in Figs $2 c$ and $7 a$ ). Thus, a forced 'oneended' invasion in mammalian cells does not preferentially trigger BIR. The major effect of a forced 'one-ended' invasion in our experiments is an $\sim 10$-fold reduction in the frequency of $\mathrm{GFP}^{+} \mathrm{RFP}^{-}$STGCs (compare STGC frequencies in Figs $2 \mathrm{c}$ and $7 \mathrm{a})$. Importantly, a gene conversion of at least $331 \mathrm{bp}$ is required to generate $\mathrm{GFP}^{+}$recombinants within the 'one-ended' reporter. The Jasin and Nickoloff labs previously measured gene conversions (GCs) within a conventional 'two-ended' reporter in mammalian cells and observed that $\sim 80 \%$ of all GCs are less 
than $58 \mathrm{bp}$ in length ${ }^{19,20}$. This suggests that the majority of STGCs occuring within the 'one-ended' reporter studied here may have been terminated prior to conversion to wtGFP and are therefore not detectable as $\mathrm{GFP}^{+}$products. Our findings are consistent with DNA fibre analysis of mammalian cells recovering from replication arrest, in which Rad51-dependent HR repair of collapsed forks-presumably mediated by one-ended invasionswas not found to be associated with replication restart ${ }^{57}$.

Importantly, a pure 'replication fork' BIR model of LTGC also fails to explain why LTGC should be relatively independent of BRCA1 in the 'two-ended' reporter (Figs. 2 and 3), but fully BRCA1-dependent in the context of the 'one-ended' reporter (Fig. 7a,b). In contrast, an SDSA model of LTGC can readily account for this difference, as it invokes a defect of HR initiation in Brcal mutant cells and, for the 'two-ended' reporter only, a defect of STGC termination by annealing (Fig. 8). These two defects will have opposing effects on the absolute frequency of LTGC only in the context of the 'two-ended' reporter and might create the appearance that LTGC is BRCA1-independent in this setting (as in Fig. 3). Notably, Rad51-mediated invasions can trigger BIR in Xenopus laevis egg extracts ${ }^{58}$ and it seems likely that a proportion of LTGCs in mammalian cells are bona fide BIR products. However, definitive analysis must await the development of an assay in mammalian cells that reliably separates BIR-mediated LTGCs from SDSA-mediated LTGCs.

A defect in annealing suggests a new mechanism underlying genomic instability associated with loss of BRCA1. In BRCA1 mutant cells, a higher proportion of HR events may escape the error-free annealing step of SDSA termination; these "functionally one-ended' invasions will be obligatorily mutagenic. Thus, the BRCA1-dependent annealing step of SDSA termination is likely an important bulwark against genomic instability and cancer. Interestingly, a recent sequencing analysis of breast cancer genomes revealed elevated frequencies of tandem gene segment duplications ${ }^{59}$. Conceivably, some of these rearrangements could be the result of inappropriate engagement of LTGC during DSB repair.

\section{Methods \\ Molecular biology and antibodies. The RFP-SCR reporter was constructed by conventional cloning methods using modified ROSA26 targeting vectors ${ }^{34}$. Expression vector for $\mathrm{h} B R C A 1$ was pcDNA3 $\beta^{60}$. siRNA SMARTpools were purchased from Dharmacon. Antibodies used were: Brcal (Santa Cruz and anti- human BRCA1 Ab MS110, 1:100), CtIP (Santa Cruz,1:50), Bard1 (Santa Cruz, 1:500), Mre11 (Novus, 1:6,000), Sirt6 (Abcam, 1:500), Exo1 (Santa Cruz, 1:500), $\beta$-actin (Abcam, 1:2,000) and influenza haemagglutinin epitope tag Ab (Santa Cruz, 1:1,000). Cells were lysed in RIPA buffer (50 mM Tris-HCl, pH 8.0, $250 \mathrm{mM}$ $\mathrm{NaCl}, 0.1 \%$ sodium dodecyl sulphate, $1 \% \mathrm{NP}-40$ with protease and phosphatase inhibitors). Extracted protein was resolved by $4-12 \%$ bis-Tris SDS-PAGE (Invitrogen) and analysed by western blotting using the antibodies described above. Supplementary Figs S8-S15 show full gel images of western blots and of the one Southern blot shown in other figures.}

Cell lines and cell culture. The Brcalfl/mut ES cell line $(11 \mathrm{CO} / 47 \mathrm{~T})$ was a kind gift from Dr Alan Ashworth ${ }^{37}$. ES cells were grown in ES medium on either MEF feeders or gelatinized plates. The RFP-SCR reporter was targeted by electroporating $2 \times 10^{7} \mathrm{BrCa}^{\mathrm{fl} / \mathrm{mut}} \mathrm{ES}$ cells with $20 \mu \mathrm{g}$ of linearized targeting vector, followed by seeding in $60-\mathrm{cm}$ plates with puroR feeders. Puromycin $\left(0.5 \mu \mathrm{g} \mathrm{ml}^{-1}\right)$ was added $24 \mathrm{~h}$ later and colonies were picked 5-7 days later. Brcal ${ }^{\mathrm{fl} / \mathrm{mut}}$ ES cells contain one mutant Brcal allele encoding a truncated gene product and a second Brcal allele (which is functionally wild type but harbours loxP sites flanking exons 22-24) that can be conditionally inactivated by Cre-mediated recombination. We generated multiple Brca1-deficient ES clones by transient adenovirus-mediated Cre expression to delete the exons 22-24. Cell line U2OS was obtained from ATCC.

Southern blotting. gDNA was extracted from confluent ES cells on six-well plates (5-10 $\times 10^{6}$ cells) using a Puregene DNA Isolation Kit (Gentra Systems). Southern blotting was performed with GFP cDNA or ROSA26 $5^{\prime}$ probe. In all, 7.5-10 $\mu$ g of gDNA was digested with the appropriate restriction enzyme and run overnight on $0.8 \%$ gel with $0.5 \times \mathrm{TBE}$ at $35 \mathrm{~V}$. The DNA was transferred overnight onto nylon membrane in $1 \mathrm{M} \mathrm{NaCl}$ and $0.4 \mathrm{M} \mathrm{NaOH}$. The membrane was prehybridized for
$30 \mathrm{~min}$ and labeled with GFP CDNA or ROSA26 $5^{\prime}$ probe overnight ${ }^{34}$. The membrane was then washed and developed by autoradiography. In all experiments, including U2OS cells containing a randomly integrated reporter, only clones containing one intact copy of the reporter were used. Supplementary Figs S8-S15 show full gel images of western blots and of the one Southern blot shown in other figures.

Recombination assays. $2 \times 10^{5}$ cells were transfected in suspension in 24-well plates with $0.5 \mu \mathrm{g}$ pcDNA3 $\beta$-myc NLS-I-SceI ${ }^{22}$ or $0.5 \mu \mathrm{g}$ control vector pcDNA3 $\beta$ using Lipofectamine 2000 (Invitrogen). GFP ${ }^{+}$and $\mathrm{GFP}^{+} \mathrm{RFP}^{+}$frequencies were measured 3 days post transfection by FACS using Becton Dickinson 5 Laser LSRII in triplicates and corrected for transfection efficiency and background events. (Transfection efficiency was measured simultaneously by parallel transfection with $0.05 \mu \mathrm{g}$ wt GFP expression vector.) Typically $\sim 2 \times 10^{5}$ total events were scored per sample. In the BRCA1 complementation experiments (Fig. 3), rescue was optimal at low levels of exogenous $B R C A 1$ expression vector $(0.1 \mu \mathrm{g}$ pcDNA3 $\beta$ $B R C A 1+0.4 \mu \mathrm{g}$ pcDNA3 $\beta$-myc NLS-I-SceI or control vector per well). For siRNA experiments, cells were transfected with $1 \mu \mathrm{l} 20 \mu \mathrm{M}$ (that is, $20 \mathrm{pmol}$ ) siRNA $+0.3 \mu \mathrm{g}$ of pcDNA3 $\beta$-myc NLS-I-SceI (or control vector) per well. In Figs 3-6, HR data represent the mean and standard error of the mean of three independent experiments. Statistical analysis was by two-tailed paired $t$-test (unknown variance).

RT-qPCR analysis. RNA was extracted using QIAGEN RNeasy Mini Kit (QIAGEN Sciences, MD). Analysis of first-strand cDNA was by Power SYBR Green RNA-toC $_{\mathrm{T}}$ 1-Step Kit (Applied Biosystems, Foster City, CA). An ABI 7300 Real time PCR System was used for RT-qPCR. Taqman probe and primer sets to genotype for Brcal were: Brcal-Exon 22-23, sense: $5^{\prime}$-TTCCGTGGTGAAGGA GCTT-3'; Brcal-Exon 22-23, antisense: $5^{\prime}$-TGGCTGCACGATCACAAC-3'; Brcal-Exon 23-24, sense: 5'-GCCTGGACAGAAGACAGCA-3'; Brca1-Exon 23-24, antisense: $5^{\prime}$-CAGTCCCACATCACAAGACG-3'. We used conventional SYBR green RT-qPCR assays of Gapdh and the siRNA-targeted gene. We used Primer 3 software (Whitehead Institute, MIT) to generate gene-specific primer sequences and confirmed use of each primer pair by melting curve analysis and gel electrophoresis. Primers used for RT-PCR: human BRCA1-sense: TCACATGAT GGGGAGTCTGA; human BRCA1-antisense: TTCCCGATAGGTTTTCCCAAA; Brcal-Exon 21-22, sense: ATGAGCTGGAGAGGATGCTG; Brcal-Exon 21-22, antisense: CTGGGCAGTTGCTGTCTTCT; Brca1-Exon 22-23, sense: GGTGC TCATCTAGTTGTGATCG; Brcal-Exon 22-23, antisense: CTGTACCAGGTA GGCATCCA; Brcal-Exon 7-8, sense: AGCCTAGGTGTCCAGCTGTC; BrcalExon 7-8, antisense: CTGCAATCACCTGGCTTAGTT; CtIP—sense: ATGGTCA AGAATCTGAACCC; CtIP—antisense: TGAGGAGGTGTCTTTGAAGCAG; Bach1-sense: ATCCGGTGTCAGAGATGTCC; Bach1-antisense: CAAGGAGT AGAGCCCGTGAG; Rap80-sense: GAAGGAAAACCCTCCTCCTG; Rap80antisense: TGTTCTTGGCCTCTCTTCGT. $m R N A$ was measured in triplicates with a standard curve generated for each gene using cDNA obtained from each sample. The expression level of target genes was normalized to internal Gapdh.

Cell cycle analysis. ES cells were pulsed with $10 \mu \mathrm{M}$ BrdU for $15 \mathrm{~min}, 48 \mathrm{~h}$ after transfection with siRNA, and fixed in $70 \%$ ethanol. BrdU was counterstained using anti-BrdU (Boehringer-Mannheim, 1:40) with a secondary FITC-conjugated rabbit anti-mouse antibody (Jackson Immuno Research, 1:50). After exposure to RNase and staining with propidium diiodide, approximately $10^{4}$ events were acquired using a Becton Dickinson 5 Laser LSR II and the results were analysed using FloJo software.

\section{References}

1. Ciccia, A. \& Elledge, S. J. The DNA damage response: making it safe to play with knives. Mol. Cell 40, 179-204 (2010).

2. Hartlerode, A. J. \& Scully, R. Mechanisms of double-strand break repair in somatic mammalian cells. Biochem. J. 423, 157-168 (2009).

3. Heller, R. C. \& Marians, K. J. Replisome assembly and the direct restart of stalled replication forks. Nat. Rev. Mol. Cell Biol. 7, 932-943 (2006).

4. Petermann, E. \& Helleday, T. Pathways of mammalian replication fork restart Nat. Rev. Mol. Cell Biol. 11, 683-687 (2010).

5. Nagaraju, G. \& Scully, R. Minding the gap: the underground functions of BRCA1 and BRCA2 at stalled replication forks. DNA Repair (Amst.) 6, 1018-1031 (2007).

6. Scully, R. \& Livingston, D. M. In search of the tumour-suppressor functions of BRCA1 and BRCA2. Nature 408, 429-432 (2000).

7. Paques, F. \& Haber, J. E. Multiple pathways of recombination induced by double-strand breaks in Saccharomyces cerevisiae. Microbiol. Mol. Biol. Rev. 63, 349-404 (1999).

8. Krogh, B. O. \& Symington, L. S. Recombination proteins in yeast. Annu. Rev. Genet. 38, 233-271 (2004).

9. San Filippo, J., Sung, P. \& Klein, H. Mechanism of eukaryotic homologous recombination. Annu. Rev. Biochem. 77, 229-257 (2008). 
10. Morrow, D. M., Connelly, C. \& Hieter, P. 'Break copy' duplication: a model for chromosome fragment formation in Saccharomyces cerevisiae. Genetics 147, 371-382 (1997).

11. Llorente, B., Smith, C. E. \& Symington, L. S. Break-induced replication: what is it and what is it for? Cell Cycle 7, 859-864 (2008).

12. Kraus, E., Leung, W. Y. \& Haber, J. E. Break-induced replication: a review and an example in budding yeast. Proc. Natl Acad. Sci. USA 98, 8255-8262 (2001).

13. Malkova, A. \& Haber, J. E. Mutations arising during repair of chromosome breaks. Annu. Rev. Genet. 46, 455-473 (2012).

14. Davis, A. P. \& Symington, L. S. RAD51-dependent break-induced replication in yeast. Mol. Cell Biol. 24, 2344-2351 (2004).

15. Malkova, A., Naylor, M. L., Yamaguchi, M., Ira, G. \& Haber, J. E. RAD51dependent break-induced replication differs in kinetics and checkpoint responses from RAD51-mediated gene conversion. Mol. Cell Biol. 25, 933-944 (2005).

16. McEachern, M. J. \& Haber, J. E. Break-induced replication and recombinational telomere elongation in yeast. Annu. Rev. Biochem. 75, 111-135 (2006).

17. Smith, C. E., Llorente, B. \& Symington, L. S. Template switching during breakinduced replication. Nature 447, 102-105 (2007).

18. Sweetser, D. B., Hough, H., Whelden, J. F., Arbuckle, M. \& Nickoloff, J. A. Fineresolution mapping of spontaneous and double-strand break-induced gene conversion tracts in Saccharomyces cerevisiae reveals reversible mitotic conversion polarity. Mol. Cell Biol. 14, 3863-3875 (1994).

19. Elliott, B., Richardson, C., Winderbaum, J., Nickoloff, J. A. \& Jasin, M. Gene conversion tracts from double-strand break repair in mammalian cells. Mol. Cell. Biol. 18, 93-101 (1998).

20. Taghian, D. G. \& Nickoloff, J. A. Chromosomal double-strand breaks induce gene conversion at high frequency in mammalian cells. Mol. Cell Biol. 17, 6386-6393 (1997)

21. Johnson, R. D. \& Jasin, M. Sister chromatid gene conversion is a prominent double-strand break repair pathway in mammalian cells. EMBO J. 19, 3398-3407 (2000)

22. Puget, N., Knowlton, M. \& Scully, R. Molecular analysis of sister chromatid recombination in mammalian cells. DNA Repair (Amst.) 4, 149-161 (2005).

23. Nagaraju, G., Odate, S., Xie, A. \& Scully, R. Differential regulation of short- and long-tract gene conversion between sister chromatids by Rad51C. Mol. Cell Biol. 26, 8075-8086 (2006).

24. Brenneman, M. A., Wagener, B. M., Miller, C. A., Allen, C. \& Nickoloff, J. A. XRCC3 controls the fidelity of homologous recombination: roles for XRCC3 in late stages of recombination. Mol. Cell 10, 387-395 (2002).

25. Nagaraju, G., Hartlerode, A., Kwok, A., Chandramouly, G. \& Scully, R. XRCC2 and XRCC3 regulate the balance between short- and long-tract gene conversion between sister chromatids. Mol. Cell Biol. 29, 4283-4294 (2009).

26. Saleh-Gohari, N. \& Helleday, T. Strand invasion involving short tract gene conversion is specifically suppressed in BRCA2-deficient hamster cells. Oncogene 23, 9136-9141 (2004).

27. Tauchi, H. et al. Nbs1 is essential for DNA repair by homologous recombination in higher vertebrate cells. Nature 420, 93-98 (2002).

28. Sartori, A. A. et al. Human CtIP promotes DNA end resection. Nature 450, 509-514 (2007).

29. Huen, M. S., Sy, S. M. \& Chen, J. BRCA1 and its toolbox for the maintenance of genome integrity. Nat. Rev. Mol. Cell Biol. 11, 138-148 (2010).

30. Greenberg, R. A. Recognition of DNA double strand breaks by the BRCA1 tumor suppressor network. Chromosoma 117, 305-317 (2008).

31. O'Donovan, P. J. \& Livingston, D. M. BRCA1 and BRCA2: breast/ovarian cancer susceptibility gene products and participants in DNA double-strand break repair. Carcinogenesis 31, 961-967 (2010).

32. Hu, Y. et al. RAP80-directed tuning of BRCA1 homologous recombination function at ionizing radiation-induced nuclear foci. Genes Dev. 25, 685-700 (2011).

33. Bunting, S. F. et al. 53BP1 inhibits homologous recombination in Brcaldeficient cells by blocking resection of DNA breaks. Cell 141, 243-254 (2010).

34. Xie, A. et al. Control of sister chromatid recombination by histone H2AX. Mol. Cell 16, 1017-1025 (2004).

35. Jasin, M. Genetic manipulation of genomes with rare-cutting endonucleases. Trends Genet. 12, 224-228 (1996).

36. Shaner, N. C. et al. Improved monomeric red, orange and yellow fluorescent proteins derived from Discosoma sp. red fluorescent protein. Nat. Biotech. 22, 1567-1572 (2004)

37. Farmer, H. et al. Targeting the DNA repair defect in BRCA mutant cells as a therapeutic strategy. Nature 434, 917-921 (2005).

38. Hakem, R. et al. The tumor suppressor gene Brcal is required for embryonic cellular proliferation in the mouse. Cell 85, 1009-1023 (1996)

39. Moynahan, M. E., Chiu, J. W., Koller, B. H. \& Jasin, M. Brcal controls homology-directed DNA repair. Mol. Cell 4, 511-518 (1999).

40. Chandler, J., Hohenstein, P., Swing, D. A., Tessarollo, L. \& Sharan, S. K. Human BRCA1 gene rescues the embryonic lethality of Brcal mutant mice. Genesis 29, 72-77 (2001).

41. Baer, R. \& Ludwig, T. The BRCA1/BARD1 heterodimer, a tumor suppressor complex with ubiquitin E3 ligase activity. Curr. Opin. Genet. Dev. 12, 86-91 (2002).
42. Drost, R. et al. BRCA1 RING function is essential for tumor suppression but dispensable for therapy resistance. Cancer Cell 20, 797-809 (2011).

43. Litman, R. et al. BACH1 is critical for homologous recombination and appears to be the Fanconi anemia gene product FANC. J. Cancer Cell 8, 255-265 (2005)

44. Coleman, K. A. \& Greenberg, R. A. The BRCA1-RAP80 complex regulates DNA repair mechanism utilization by restricting end resection. J. Biol. Chem. 286, 13669-13680 (2011)

45. Adamson, B., Smogorzewska, A., Sigoillot, F. D., King, R. W. \& Elledge, S. J. A genome-wide homologous recombination screen identifies the RNA-binding protein RBMX as a component of the DNA-damage response. Nat. Cell Biol. 14, 318-328 (2012).

46. Wang, B. et al. Abraxas and RAP 80 form a BRCA1 protein complex required for the DNA damage response. Science 316, 1194-1198 (2007).

47. Kaidi, A., Weinert, B. T., Choudhary, C. \& Jackson, S. P. Human SIRT6 promotes DNA end resection through CtIP deacetylation. Science 329, 1348-1353 (2010)

48. Xie, A. et al. Distinct roles of chromatin-associated proteins MDC1 and 53BP1 in mammalian double-strand break repair. Mol. Cell 28, 1045-1057 (2007).

49. Yun, M. H. \& Hiom, K. CtIP-BRCA1 modulates the choice of DNA doublestrand-break repair pathway throughout the cell cycle. Nature 459, 460-463 (2009).

50. Westmoreland, J. W. \& Resnick, M. A. Coincident resection at both ends of random, gamma-induced double-strand breaks requires MRX (MRN), Sae2 (Ctp1), and Mre11-nuclease. PLoS Genet. 9, e1003420 (2013).

51. Helmink, B. A. et al. H2AX prevents CtIP-mediated DNA end resection and aberrant repair in G1-phase lymphocytes. Nature 469, 245-249 (2011).

52. Jain, S. et al. A recombination execution checkpoint regulates the choice of homologous recombination pathway during DNA double-strand break repair. Genes Dev. 23, 291-303 (2009).

53. Kane, D. P., Shusterman, M., Rong, Y. \& McVey, M. Competition between replicative and translesion polymerases during homologous recombination repair in Drosophila. PLoS Genet. 8, e1002659 (2012).

54. Lydeard, J. R. et al. Break-induced replication requires all essential DNA replication factors except those specific for pre-RC assembly. Genes Dev. 24, 1133-1144 (2010).

55. Ira, G. \& Haber, J. E. Characterization of RAD51-independent break-induced replication that acts preferentially with short homologous sequences. Mol. Cell Biol. 22, 6384-6392 (2002).

56. Hastings, P. J., Ira, G. \& Lupski, J. R. A microhomology-mediated breakinduced replication model for the origin of human copy number variation. PLoS Genet. 5, e1000327 (2009).

57. Petermann, E., Orta, M. L., Issaeva, N., Schultz, N. \& Helleday, T. Hydroxyurea-stalled replication forks become progressively inactivated and require two different RAD51-mediated pathways for restart and repair. Mol. Cell 37, 492-502 (2010).

58. Hashimoto, Y., Puddu, F. \& Costanzo, V. RAD51- and MRE11-dependent reassembly of uncoupled CMG helicase complex at collapsed replication forks. Nat. Struct. Mol. Biol. 19, 17-24 (2012).

59. Stephens, P. J. et al. Complex landscapes of somatic rearrangement in human breast cancer genomes. Nature 462, 1005-1010 (2009).

60. Scully, R. et al. Association of BRCA1 with Rad51 in mitotic and meiotic cells Cell 88, 265-275 (1997).

\section{Acknowledgements}

We thank Dr Alan Ashworth for the Brcal $1^{\mathrm{f} / \text { mut }}$ ES cell line and Drs Richard Baer and Jagesh Shah for plasmids and antibodies. We thank Drs Michael Resnick and Anna Malkova for communicating the results of unpublished experiments, Drs Jim Haber, Lorraine Symington and Steve Jackson, as well as members of the Scully lab for valuable comments and critique. This work was supported by NIH grants CA095175 and GM073894 (to R.S.), T32 Training Grant in Cancer Biology CA081156 and an ACS postdoctoral fellowship (to N.A.W.).

\section{Author contributions}

G.C., A.K., B.H., N.A.W. and A.X. performed the experiments. G.C. and R.S. designed the new HR reporters. G.C., N.A.W. and R.S. wrote the paper.

\section{Additional information}

Supplementary Information accompanies this paper at http://www.nature.com/ naturecommunications

Competing financial interests: The authors declare no competing financial interests

Reprints and permission information is available online at http://npg.nature.com/ reprintsandpermissions/

How to cite this article: Chandramouly, G. et al. BRCA1 and CtIP suppress long-tract gene conversion between sister chromatids. Nat. Commun. 4:2404 doi: $10.1038 /$ ncomms3404 (2013). 